\title{
A NEW METHOD OF REDUCTION FOR DIFFRACTION SPECTRA OBSERVATIONS.
}

By Emit Rosenbera, M. D., New York.

The apparatus employed is one of those ordinary two-tube spectroscopes, provided with a simple photograph scale placed in the eyepiece of the observing telescope, and presenting divisions from 0 to 160 (actually from 10 to 160.) Though allowing only approximate determinations, such a scale is found, in certain respects,' to answer our purpose better than other descriptions of measuring apparatus.

The necessity of communicating spectrum observations by referring them to wavelengths, instead of to an arbitrary scale, has long been established. The arbitrary, individual scale of a prismatic spectrum is easily converted into the rational scale of the Normal Spectrum by various methods. Though these do not here concern us, we may call the attention of observers to a table, computed by Professor Vierordt ("Die quantitative Spectralanalyse, Tübingen," 1876, page 104), which we have found very useful for this purpose.

The diffraction-grating gives the desired wavelengths directly by the well known proceeding, which ought certainly not to be replaced by a less exact method. Setting aside, however, the circumstance that not every spectroscopist is provided with the necessary measuring apparatus, there are other obvious reasons for the employment of convenient reduction-methods. Spectroscopical work is not confined to occasional investigations of the discoverer, who will prefer direct measurement, but the accumulated facts and results find eager students intent upon their acquisition. The study of established facts requires occasionally other methods than the original investigator was obliged to employ. Even the latter will, in timeconsuming examinations, avail himself of reduction methods, if they permit exact determination. Moreover, the author believes that certain features of the method offered in this paper will even be profitably employed in combination with direct measurement.

The essential point for a convenient reduction-method is, to use a position of the grating, which should yield a simple and-for all regions of the spectrum-equal ratio of distances of the Fraunhofer lines in reference to a standard scale.

As such we adopt Angström's tenth-metre scale. 
Searching for such a simple ratio with Mr. L. M. Rutherfurd's transparent 6481-line grating (which may be called $R_{a}$ ), the author found for the position of minimum deviation,

All distances of Spectrum I at the ratio 1:20 of Angström's,

$\begin{array}{llllllll}\text { " " " } & \text { " } & \text { II } & \text { " } & 2: 20 & \text { "6 } & \\ & \text { III } & \text { " } & 3: 20 & \text { " } & \text { etc. }\end{array}$

Starting from this observation, the following relations were established for a series of diffraction-gratings :

\section{TABLE I.}

\begin{tabular}{|c|c|c|c|c|c|c|c|c|c|c|}
\hline \multirow{2}{*}{\multicolumn{2}{|c|}{$\begin{array}{c}\text { Designation of } \\
\text { Grating. }\end{array}$}} & \multirow{2}{*}{$\mid \begin{array}{c}\text { Number of } \\
\text { intervals } \\
\text { per English } \\
\text { inch. }\end{array}$} & \multicolumn{2}{|c|}{$\begin{array}{l}\text { Multiples } \\
\text { of }\end{array}$} & \multicolumn{6}{|c|}{$\begin{array}{l}\text { Relation of Distances (at min. dev. } \\
\text { position) to tenth-metre scale, } \\
\text { for Spectrum. }\end{array}$} \\
\hline & & & 540 & 2160 & I. & II. & III. & IV. & V. & VI. \\
\hline \multirow{5}{*}{ 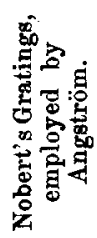 } & $\mathbf{A}_{i j i}$ & 2160 & 4 & 1 & $\frac{1}{60}$ & $\frac{1}{30}$ & $\frac{1}{20}$ & $\frac{1}{15}$ & $\frac{1}{12}$ & $\frac{1}{10}$ \\
\hline & $A_{v}$ & 2700 & 5 & $1 \frac{1}{4}$ & $\frac{1}{48}$ & $\frac{1}{24}$ & $\frac{1}{16}$ & $\frac{1}{12}$ & $\frac{5}{48}$ & $\frac{1}{8}$ \\
\hline & $\mathbf{A}_{\mathrm{ii}}$ & 3240 & 6 & $1 \frac{1}{2}$ & $\frac{1}{40}$ & $\frac{1}{20}$ & $\frac{3}{40}$ & $\frac{1}{10}$ & $\frac{1}{8}$ & $\frac{3}{20}$ \\
\hline & $\mathbf{A}_{\mathrm{iv}}$ & 4320 & 8 & 2 & $\frac{1}{30}$ & $\frac{1}{15}$ & $\frac{1}{10}$ & $\frac{2}{15}$ & $\frac{1}{6}$ & $\frac{1}{5}$ \\
\hline & $A_{i}$ & 5400 & 10 & $2 \frac{1}{2}$ & $\frac{1}{24}$ & $\frac{1}{12}$ & $\frac{1}{8}$ & $\frac{1}{6}$ & $\frac{5}{24}$ & $\frac{1}{4}$ \\
\hline \multirow{3}{*}{ 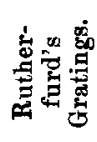 } & $\mathbf{R}_{\mathrm{a}}$ & 6480 & 12 & 3 & $\frac{1}{20}$ & $\frac{1}{10}$ & $\frac{3}{20}$ & $\frac{1}{5}$ & $\frac{1}{4}$ & $\frac{3}{10}$ \\
\hline & $\mathbf{R}_{\mathrm{b}}$ & 8640 & 16 & 4 & $\frac{1}{15}$ & $\frac{2}{15}$ & $\frac{1}{5}$ & $\frac{4}{15}$ & $\frac{1}{3}$ & $\frac{2}{5}$ \\
\hline & $\mathbf{R}_{\mathrm{o}}$ & 17280 & 32 & 8 & $\frac{2}{15}$ & $\frac{4}{15}$ & $\frac{2}{5}$ & $\frac{8}{15}$ & $\frac{2}{3}$ & $\frac{4}{5}$ \\
\hline
\end{tabular}

A grating of 129,600 intervals per inch would, theoretically, answer the tenth-metre scale, though actually no spectrum could be obtained from such a plate. This number may, however, be employed for finding the relation of any grating to Angström's scale. Thus, f. i., 8100 intervals per inch would present the ratio $\frac{1}{16}$ for Sp. I; a grating with a ratio of $\frac{1}{10} A$ for Sp. I, must have 12,960 intervals per inch.

The coefficients, given in Table I, have been verified for Sp. I and II of the following gratings:

$$
\begin{aligned}
& A_{v} \text { (a photograph), } \\
& A_{i v} \text { (ruled on glass, by Mr. Rutherfurd), } \\
& R_{a} \text { (a photograph), } \\
& R_{b}\{\text { (ruled on speculum metal). } \\
& R_{c}(\text { rulut }
\end{aligned}
$$


These coefficients will serve various purposes:

1. For finding the correct position of minimum deviation of any grating belonging to the series, through the distances of the Fraunhofer lines, for Sp. I.

2. For determining rational scale-numbers directly from known wavelengths.

3. For determining wavelengths from observed scale-numbers.

4. For finding the number of intervals or lines of a grating, etc.

The reduction-method, based upon this definite relation, will recommend itself by its simplicity.

The grating being placed, in the usual way, at the presumptive position of minimum deviation, the latter is approximately corrected (by means of the photograph scale) by equalizing the ratio of distances of the Fraunhofer or metal lines, in reference to A's distance, according to the coefficient for Sp. I.

Thus, $R_{n}$ would be adjusted to such a position, that, $f$. $i$, A's distance $\mathrm{C}-\mathrm{D}^{\prime \prime}=667^{\text {ronth motre }}$ would be reduced to $33^{\text {div. }}, 3$,

\begin{tabular}{|c|c|c|c|c|}
\hline $\mathrm{D}^{\prime \prime}-\mathrm{E}=626$ & “6 & “6 & 66 & $31^{\text {div. }, 3,}$ \\
\hline$E-b^{\prime}=86$ & “ & 16 & 66 & 4 div., 3 , \\
\hline $\mathrm{Li} \alpha-\mathrm{Na}=813$ & “ & 6 & 66 & div., 6 , \\
\hline $\mathrm{Na}-\mathrm{Th}=543$ & 6 & 66 & “6 & , et \\
\hline
\end{tabular}

A continuous, rational scale is then formed, the scale-numbers being obtained directly from the known wavelengths, by multiplying $\lambda$ by the respective coefficient, in this instance, by $\frac{1}{20}$.

The wavelength is found from the observed scale-number, by multiplying the latter by the inverted coefficient, in this instance, by 20 .

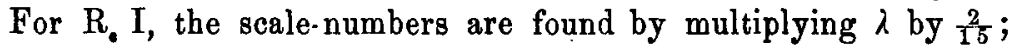
the wavelength, by multiplying the observed scale-number by $\frac{15}{2}$.

It is scarcely necessary to remark, that for determination of unknown wavelengths, a more delicate measuring apparatus (micrometer screw or similar means ${ }^{i}$ ) will have to replace the photograph scale, after this has done its work.

Simple, as this method is, it was reached only after and through another more complicated one, which must be mentioned, though not recommended for practical application.

This latter method gives a discontinuous scale, each spectrum being observed in five separate strips, each being destined for a

'Prof. Rood's micrometer eye-piece, (Silliman's Journal, 1873.) 
certain number of rays (999 tenth-metre actually fitting the scale from 0 to 160 ).

The first strip for rays of 3000 to 3999 tenth metre,

$\begin{array}{lllll}\text { " second } & \text { " } & \text { " } & 4000 \text { to } 4999 & \text { " } \\ \text { " third } & \text { " } & \text { " } & 5000 \text { to } 5999 & \text { " } \\ \text { " fourth } & \text { " } & \text { " } & 6000 \text { to } 6999 & \text { " } \\ \text { " fifth } & \text { " } & \text { " } 7000 \text { to } 7604 & \text { " }\end{array}$

These strips are numbered respectively $3,4,5,6$ and 7 . An incidental feature of this method is the separation of $\lambda$ in two parts, viz.:

1. The first figure.

2. The balance, which is designated " $l$." Thus, of $\lambda 6705$, 4 , we call " $l$ " the number 705,4 . The rational scale-number is here found from the known wavelength by multiplying $l$ by the proper coefficient, and placing the number found in the strip indicated by the first figure of the wavelength.

Spectrum $I$ of $R_{\mathrm{a}}$, arranged in 5 parts would accordingly read as follows:

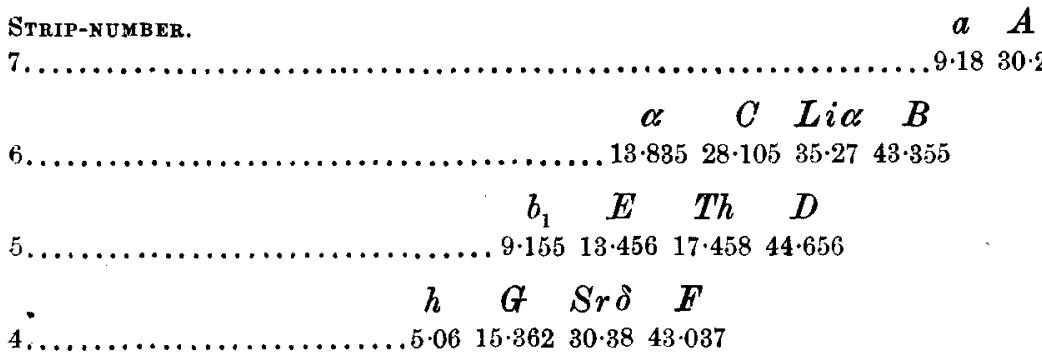

$\begin{array}{llllllllll}R & Q & P & O & N & M & L & H_{1} & H\end{array}$

$3 \ldots . .8 \cdot 8 \quad 14 \cdot 3 \quad 18 \quad 22 \quad 29 \quad 36 \cdot 45 \quad 40 \cdot 95 \quad 46 \cdot 65 \quad 48 \cdot 405$

The wavelength is here found, by multiplying the observed scalenumber by the inverted coefficient. The product is " $l$," to which the strip-number has to be prefixed as 1st figure.

A scale-number would, by this method, be incomplete without adding the strip-number in brackets; e. $g ., 35,27\left(R_{2} I, 6\right)=$ scalenumber for $L i \alpha$.

We now return to the simple method:

By an observation made upon the two gratings, $A_{i v}$ and $A_{v}$, the length of the first dark space, for any grating of the series, was directly determined. With these two gratings, the dispersion-in the minimum deviation position-is very small, showing the Normal simultaneously with $H_{1}$ and several other Fraunhofer lines. 
The scale-numbers for $\mathrm{H}_{1}$-as calculated from $\lambda \mathrm{H}_{1}$-were found by observation to correspond with the distance from the Normal in actual divisions of the scale.

The same holds good for Angström's wavelengths, which represent distances from an ideal Normal in divisions of his scale, in tenthmetres.

Direct observation showed the length of the first dark space for $A_{i v}$, to be about 100 div., for $A_{v}$ about 63 div.

The scale-number: $H_{i}$ for $A_{i r}$ was observed at about 131, for $A_{r}$ at about 82 (calculated respectively 131,1 and $81,9$. )

From comparing the distance $\mathrm{H}_{1}-\operatorname{Normal}\left(A_{\mathrm{iv}} I\right)$ with the corresponding scale-number $\mathrm{H}_{1}$ in the five strip arrangement: $31,1-$ the first dark space was concluded to measure here exactly 100 div., the interval between 100 and 131, 1 being evidently filled by the ultraviolet spectrum.

In the same way the first dark space for $A_{v} I$ was determined as measuring 62.5 div.

By means of the "reference numbers," the corresponding relations for the entire series ${ }^{1}$ were then easily found, as detailed in Table II.

TABLE II.

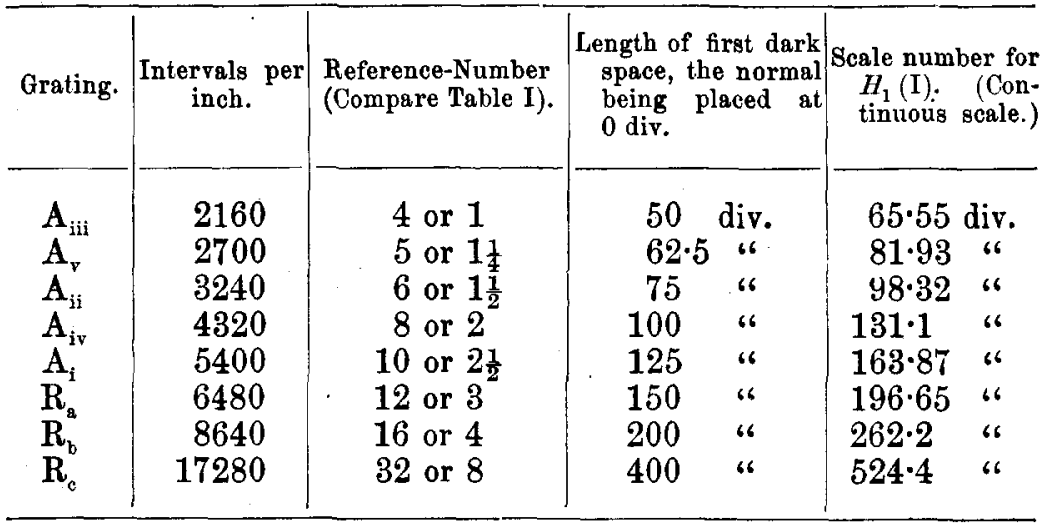

The dark space of Angström's spectrum would measure 3000 div. of his scale, 933 div: belonging to his ultraviolet.

There is another result of this reduction-method, which the author wishes to submit to the indulgent reader.

\footnotetext{
'Only those gratings of the "series," which actually exist, are specified in this paper.
} 
By comparing our scale-number for

$$
\begin{array}{r}
\mathrm{D}(\mathrm{M}), \mathbf{R}_{\mathrm{a}} \mathbf{I} \\
294 \text { dir., } 60625,
\end{array}
$$

with its directly measured angle of deviation from the Normal,

$$
8^{\circ} 39^{\prime}=519^{\prime} \text {, }
$$

one division of our scale was found to be equivalent to $1^{\prime}, 762$.

This holds good for any grating of the series, and for any order of spectrum.

We were thus enahled to compute in the easiest manner the "angles" for all the principal Fraunbofer lines for a number of gratings.

By employing the above-mentioned number, the angle for the $D$ line $\left(\mathrm{R}_{\mathrm{a}} \mathrm{I}\right)$ was computed as $8^{\circ} 39^{\prime} 5^{\prime \prime}, 7$.

The measured angle $8^{\circ} 39^{\prime}$ being certainly correct, as far as the division of the circle allowed, we might be satisfied with this base for our computations.

We believe, however, to have found a reason for changing the equivalent $1^{\prime}, 762$ for $1^{\prime}, 8$, which would make the specified angle

$$
8^{\circ} 50^{\prime} 17^{\prime \prime}, 4 \text {. }
$$

We may be permitted to state the considerations upon which our reasoning is based.

The angles corresponding to Angström's wavelengths may be calculated in the same way, as indicated above for the spectra of actually existing gratings. We find it, in fact, convenient to calculate these phantom angles from the wavelengths, and to reduce them according to the coefficients for the various gratings.

Employing for this purpose the number 1, 762, we find the angle corresponding to wavelength

$$
\begin{aligned}
& 1000=29^{\circ} 22^{\prime} \text {, while by employing } 1,8=30^{\circ} \text {, } \\
& 2000=58^{\circ} 44^{\prime}, \text { " " " " " }=60^{\circ} \text {, } \\
& 3000=88^{\circ} 06^{\prime}, \quad \text { " " " " " " }=90^{\circ} \text {, } \\
& 4000=117^{\circ} 28^{\prime} \text {, " " " " " " }=120^{\circ} \text {, } \\
& 5000=146^{\circ} 50^{\prime}, \quad \text { " " " " " }=150^{\circ} \text {, } \\
& 6000=176^{\circ} 12^{\prime}, \quad \text { " " " " " }=180^{\circ} \text {, } \\
& 7000=205^{\circ} 34^{\prime}, \quad " \quad " \quad " \quad "=210^{\circ} \text {. }
\end{aligned}
$$

It is this striking regularity and harmony which leads us, at least, to doubt the correctness of the usual measurements. Instrumental means for investigating and deciding this matter are not at our disposal. 